INPLASY

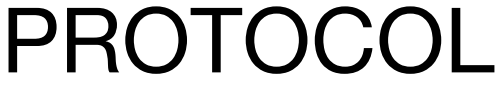

To cite: Gao et al.

Hyperuricemia increases the risk of atrial fibrillation: a systematic review and metaanalysis. Inplasy protocol 2021120092. doi:

10.37766/inplasy2021.12.0092

Received: 21 December 2021

Published: 21 December 2021

Corresponding author:

Zheng Gao

2966526665@qq.com

Author Affiliation:

Department of Second

Hospital of Hebei Medical

University, P. R. China.

Support: There are no funding sources.

Review Stage at time of this submission: Formal screening of search results against eligibility criteria.

Conflicts of interest:

None declared.

\section{Hyperuricemia increases the risk of atrial fibrillation: a systematic review and meta-analysis}

Gao, X1; Shi, X2; Xu, W3; Su, X4; Guan, Z5; Guo, N6; Ma, H².

Review question / Objective: Association between HUA and AF remains unclear, for which the present meta-analysis will systematically review clinical evidence and may give suggestions for therapy of those AF patients with gout or HUA.

Condition being studied: Hyperuricacidemia (HUA) is a metabolic disease caused by purine metabolism and imbalance of uric acid production and excretion, with a uric acid level $>7.0 \mathrm{mg} / \mathrm{dL}$ in men and $>5.7 \mathrm{mg} / \mathrm{dL}$ in women. Atrial fibrillation is known as a common cardiac disease. Information sources: PubMed, Clinical trial, Embase database, Cochrane Library, China national knowledge infrastructure (CNKI), Epigraph DB (https://epigraphdb.org/), DigiZeitschriften (http://www.digizeitschriften.de/) and Web of Science. We will ask authors for literatures if possible.

INPLASY registration number: This protocol was registered with the International Platform of Registered Systematic Review and Meta-Analysis Protocols (INPLASY) on 21 December 2021 and was last updated on 21 December 2021 (registration number INPLASY2021120092).

\section{INTRODUCTION}

Review question / Objective: Association between HUA and AF remains unclear, for which the present meta-analysis will systematically review clinical evidence and may give suggestions for therapy of those AF patients with gout or HUA.

Condition being studied: Hyperuricacidemia (HUA) is a metabolic disease caused by purine metabolism and imbalance of uric acid production and 
excretion, with a uric acid level $>7.0 \mathrm{mg} / \mathrm{dL}$ in men and $>5.7 \mathrm{mg} / \mathrm{dL}$ in women. Atrial fibrillation is known as a common cardiac disease.

\section{METHODS}

Participant or population: General population.

Intervention: We will include observational studies, including retrospective cohort studies, cross-sectional studies, and prospective cohort studies. The exposure we will study was mainly hyperuricemia (HUA).

Comparator: We will include observational studies, including retrospective cohort studies, cross-sectional studies, and prospective cohort studies. The comparators will be those individuals without hyperuricemia and with or not atrial fibrillation.

Study designs to be included: We will include representative cohort studies, cross-sectional studies and prospective cohort studies.

Eligibility criteria: We will exclude studies that may lead to an incredible conclusion. We will include cross-sectional, retrospective and prospective cohort studies. Randomized controlled trials will be excluded. The reason is that randomized controlled trials are too rare to lead to independently certain results, as introduced later. And difference in study designing will bring uncertainty to the evidence provided in this study.Study type should be clearly defined. Cross-sectional studies should clearly introduce the sources of epidemiological data. Sources as public health databases, hospital medical records, or insurance agencies will be acceptable. The number of participants should be sufficient to avoid contingency. Any study of participants less than $\mathbf{2 0 0}$ will be excluded. Studies will be excluded if with too many complications unrelated to AF enrolled. We will include only studies that all the follow-up work completed to ensure the whole data is available. Criteria of hyperluricacid or AF should be defined clearly, which should be consistent with the universal knowledge. Studies get "Critical risk" in overall will be excluded. Retrospective studies reporting association between AF and HUA will be included. Participants should be defined with hyperuricemia directly. Case reports, protocols or trials with incomplete followup will be excluded.

Information sources: PubMed, Clinical trial, Embase database, Cochrane Library, China national knowledge infrastructure (CNKI), Epigraph DB (https://epigraphdb.org/), DigiZeits chriften (http: / / www.digizeitschriften.de/) and Web of Science. We will ask authors for literatures if possible.

Main outcome(s): To evaluate the proportion of atrial fibrillation in people with and without hyperuricemia.

Quality assessment / Risk of bias analysis: Quality evaluation will be operated by $\mathbf{R}$ version 4.1.2, using the revised Cochrane risk-of-bias tool for randomized trials (ROB2). One reviewer (G.Z.) will evaluate all studies included in seven domains: 1 . Bias due to confounding 2. Bias due to selection of participants 3 . Bias in classification of interventions 4. Bias due to deviations from intended interventions 5 . Bias due to missing data 6 . Bias in measurement of outcomes 7. Bias in selection of the reported result. Risk of each study will be labelled as "Low risk", "Moderate risk", "Serious risk" and "Critical risk".

Strategy of data synthesis: Data synthesis will be operated on $R$ version 4.1.2, using the package "meta" (R Project for Statistical Computing) (R Core Team. R: a language and environment for statistical computing. Vienna R Foundation for Statistical Computing; 2019. https:// www.R-project.org). Risk ratio (RR) and its $95 \%$ confidence interval will be used to evaluate the proportion of atrial fibrillation in people with and without hyperuricemia. Studies will be sub-grouped and regressed to detect possible sources of heterogeneity and potential influencing factors of $\mathrm{AF}$ 
proportion. All statistical effects will be calculated in a random effect model, with a two-tailed $a=0.05$ set as the statistical significance. The Cochrane's $Q$ test and $I^{2}$ will be calculated as measures of heterogeneity. $I^{2}$ values of $25 \%, 50 \%, 75 \%$ will be thought to indicate a low, moderate or high heterogeneity.

Subgroup analysis: Important covariates such as race, country or study design will be used as a classification basis for subgroup analysis. Subgroup analysis is intended to test heterogeneity and factors that may affect results.

Sensitivity analysis: Sensitivity analysis will be performed using the function "metainf", package "meta" on $R$ version 4.1.2.

Country(ies) involved: China.

Keywords: hyperuricacidemia; atrial fibrillation; prevalence.

Contributions of each author:

Author 1 - Zheng Gao.

Email: 2966526665@qq.com

Author 2 - He-Kai Shi.

Email: 2534325742@qq.com

Author 3 - Wei Xu.

Email: xdfxvwei@163.com

Author 4 - Xiuxiu Su.

Email: 504674287@qq.com

Author 5 - Zhengzhao Guan.

Email: guanzhengzhao@gmail.com

Author 6 - Nuojin Guo.

Email: 3127223640@qq.com

Author 7 - Huijie Ma.

Email: huijiema@hebmu.edu.cn 